\title{
Reply to Gaertner, K. and Frass, M.
}

\author{
Matteo Monami ${ }^{1} \mathbb{D} \cdot$ Antonio Silverii $^{1} \cdot$ Edoardo Mannucci $^{1}$
}

Received: 21 October 2018 / Accepted: 25 October 2018 / Published online: 24 November 2018

(c) Springer-Verlag Italia S.r.l., part of Springer Nature 2018

We read with interest the comment to our prior letter $[1,2]$. The reported references show quite clearly that authors of studies on homeopathy do not feel the need to share their results with the scientific community in the usual patterns. In fact, of the alleged eight studies, two (\#4 and 6) are not papers, whereas one (\#11) is not indexed on any of the databases used for our search; in addition, this latter paper and the journal on which it is published cannot be identified with a common search engine.

Of the remaining five studies, two are not randomized (\#5 and 7), and two enrolled both overweight and obese patients $(\# 9,10)$; of the latter, one (\#10) is written in a language other than English, whereas our search was limited to English language papers. Finally, the last paper (\#8), also written in a language other than English, reported that a homeopathic treatment had no effect on body weight or waist circumference.

Overall, we do not think that these additional papers can modify in any way the conclusion of our letter. In fact, although there is one pilot trial (out of the boundaries of our search strategy) showing that homeopathy has no effect on body weight, we feel that this evidence is not sufficient to rule out the possibility that this approach has some biological effects (either beneficial or harmful) in patients with diabetes and/or obesity.

\section{Compliance with ethical standards}

Conflict of interest Matteo Monami has received speaking fees from Bristol Myers Squibb, Eli-Lilly, Merck, Novonordisk, Merck, and Takeda, and research grants from Bristol Myers Squibb. Antonio Silverii has no conflict of interests. Edoardo Mannucci has received consultancy fees from Merck and Novartis, speaking fees from Astra Zeneca, Bristol Myers Squibb, Merck, and Novartis, and research grants from Merck, Novartis, and Takeda.

Ethical approval This article does not contain any studies with human participants performed by any of the authors.

Informed consent For this type of study, formal consent is not required.

\section{Reference}

1. Gaertner K, Frass M (2018) Comment on Monami et al. "Alternative treatment or alternative to treatment? A systematic review of randomized trials on homeopathic preparations for diabetes and obesity". Acta Diabetologica. https://doi.org/10.1007/s0059 2-018-1247-3

2. Monami M, Silverii A, Mannucci E (2018) Alternative treatment or alternative to treatment? A systematic review of randomized trials on homeopathic preparations for diabetes and obesity. Acta Diabetol. https://doi.org/10.1007/s00592-018-1235-7

Managed by Massimo Porta.

This reply refers to the comment available online at https://doi. org/10.1007/s00592-018-1247-3.

Matteo Monami

mmonami11@gmail.com

1 Diabetology, Azienda Ospedaliero-Universitaria Careggi and University of Florence, Florence, Italy 